\title{
Novel EELS Experiments in the Newly Opened Monochromated Regime
}

\author{
Jordan A. Hachtel ${ }^{1}$, Jacob R. Jokisaari ${ }^{2}$, Robert F. Klie ${ }^{2}$, and Juan Carlos Idrobo ${ }^{1}$ \\ 1. Center for Nanophase Materials Sciences, Oak Ridge National Laboratory, Oak Ridge, TN USA. \\ 2. Department of Physics, University of Illinois at Chicago, Chicago, IL USA.
}

Electron energy loss spectroscopy (EELS) has long been a highly utilized spectroscopy method within the scanning transmission electron microscopy (STEM) community due to its ability to correlate spectroscopic data with an atomic sized probe. However, the precision and energy resolution of a standard EELS measurement is limited by the distribution of energies that arise from the field emission of the electron gun.

The width of this distribution is observed in the zero-loss peak (ZLP) of the EEL spectrum, which contains all the electrons that scatter elastically within the sample. The energy resolution is most commonly measured as the full-width at half-maximum (FWHM) of the ZLP, which is typically 300 $\mathrm{meV}$ for a cold field emission gun (cFEG) and is closer to $\sim 700 \mathrm{meV}$ for thermionic and Schottky field emission guns [1,2]. Beyond the energy resolution, the ZLP tails extend well into the visible spectral regime (1-2 eV), creating "background" that can obscure weak signals. For many EELS features, such as core-loss edges and bulk plasmons, the limitation imposed by ZLP tails and energy resolution are negligible. However, for optical excitations in the telecomm/near-infrared (IR) regime, higher background levels and lower energy resolution make analysis extremely challenging. For vibrational excitations in the mid-IR to far-IR, the levels imposed are prohibitive, which until recently has prevented EELS from fully exploiting opportunities in this energy regime.

The EELS energy resolution can be improved via monochromation, and recent breakthroughs in STEM monochromation have enabled an energy resolution improvement of over an order of magnitude, and dramatically reduced the ZLP tail background in the IR [3]. Figure 1a shows the ZLPs for different EEL spectra without monochromation (black) and with increasing levels of monochromation (green). The improvement in energy resolution is observed in the ZLP-FWHM, which decreases from $287 \mathrm{meV}$ to 22 $\mathrm{meV}$ with higher levels of monochromation. Even for low levels of monochromation (FWHM=259 $\mathrm{meV}$ ), the improvement in the ZLP tail background is obvious. Figure $1 \mathrm{~b}$ shows the same ZLPs as those shown in Fig. 1a, but here the ZLPs are plotted on a log-plot to better quantify the background reduction. For the highest levels of monochromation, the ZLP tail is reduced by three orders of magnitude for the majority of the IR, and reduced to less than $1 / 1000^{\text {th }}$ of its maximum intensity at energy losses less than $100 \mathrm{meV}$.

Monochromated electron probe analysis in the near-IR/telecomm (400-1600 meV) goes from being difficult to straightforward, and analysis in the mid-IR and far-IR regime $(<400 \mathrm{meV})$ becomes possible. While these regimes have been accessible for some time with techniques such as infrared and Raman spectroscopy, they are now accessible in a STEM with an atomic-sized electron probe.

Here, we employ a Nion aberration-corrected high energy resolution monochromated EELS-STEM $\left(\right.$ HERMES $^{\mathrm{TM}}$ ) to probe mid-IR excitations such as plasmons and phonons in complex nanostructures, combining a high level of both spatial and spectral resolution. Additionally, we utilize 'aloof' excitation EELS to perform vibrational spectroscopy on highly beam-sensitive materials and distinguish between 
the phonon modes of different isotopes. Lastly, we demonstrate how the monochromator can be used to manipulate the electron beam to perform advanced STEM experiments and examine new magnetic and optical phenomena.

\section{References:}

[1] K Kimoto \& Y Matsui, J. Microsc. 208 (2002), p. 224.

[2] RF Egerton, Rep. Prog. Phys. 72 (2009), p. 016502.

[3] OL Krivanek et al., Nature 514 (2014), p. 13870.

[4] Research supported by ORNL's Center for Nanophase Materials Sciences, which is a U.S. Department of Energy Office of Science User Facility and by ORNL's Laboratory Directed Research and Development Program, managed by UT-Battelle, LLC, for the U.S. Department of Energy.
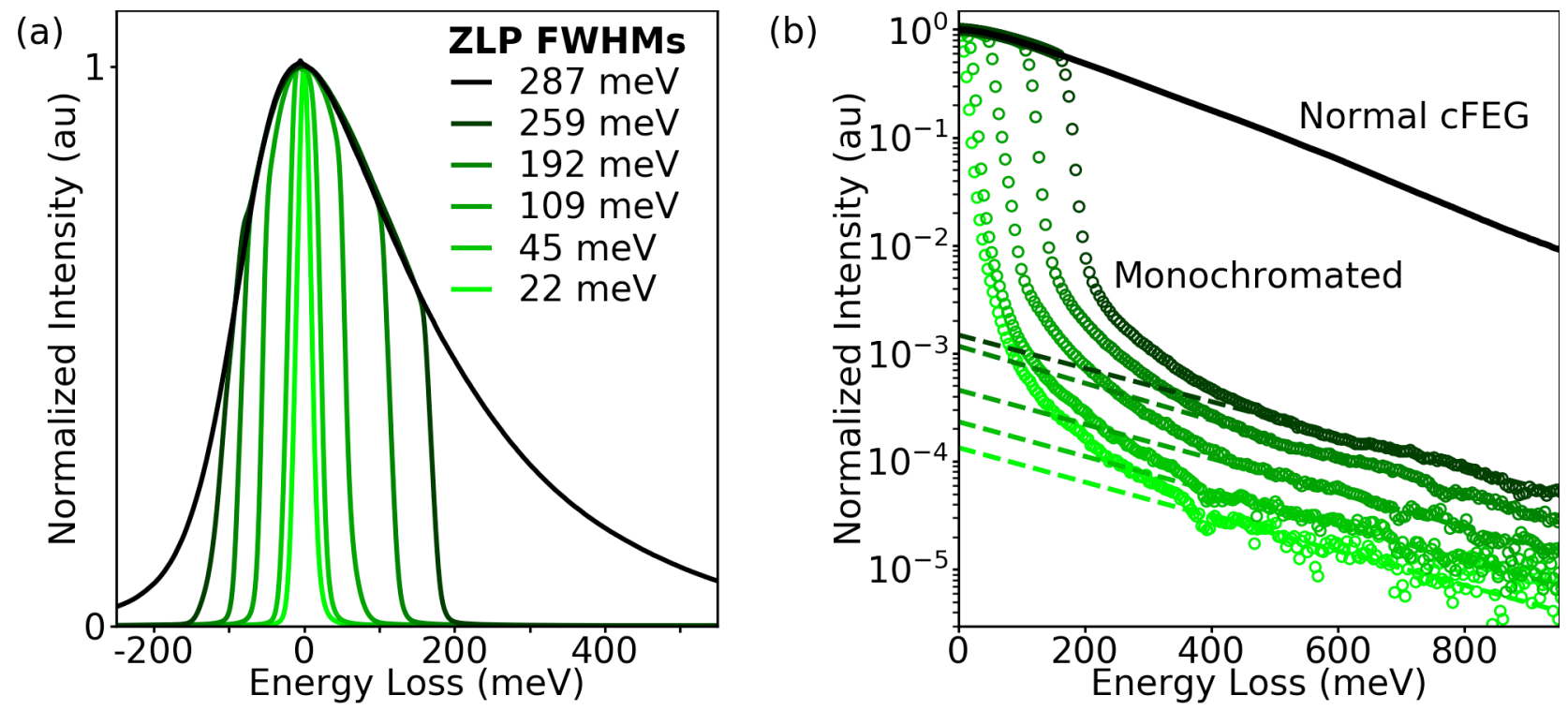

Figure 1. (a) EELS ZLPs at varying degrees of monochromation, demonstrating an order of magnitude energy resolution improvement in modern STEMs. (b) Log plots of ZLP tails from (a), demonstrating several orders of magnitude background reduction throughout IR regime, opening up new opportunities for EELS experiments. 